Supporting information for

\title{
Synthesis of Biladienone and Bilatrienone by Coupled Oxidation of Tetraarylporphyrins
}

Naomi Asano, Sayo Uemura, Tomoya Kinugawa, Hiroaki Akasaka, and Tadashi Mizutani

Department of Molecular Science and Technology, Faculty of Engineering, Doshisha University,

Tatara-Miyakotani, Kyotanabe, Kyoto 610-0321 Japan

Table of Contents:

Page S1

Table of Contents

Page S2

${ }^{1} \mathrm{H}$ NMR of $\mathbf{2 a}$

Page S3

${ }^{13} \mathrm{C}$ NMR of $\mathbf{2 a}$

Page S4

${ }^{1} \mathrm{H}$ NMR of $\mathbf{2 b}$

Page S5

${ }^{13} \mathrm{C}$ NMR of $\mathbf{2 b}$

Page S6

${ }^{1} \mathrm{H}$ NMR of 2c

Page S7

${ }^{1} \mathrm{H}-{ }^{1} \mathrm{H}$ COSY of $\mathbf{2 c}$

Page S8

ROESY of $\mathbf{2 c}$

Page S9

${ }^{13} \mathrm{C}$ NMR of $2 \mathrm{c}$

Page S10

HMBC of 2c

Page S11

${ }^{1} \mathrm{H}$ NMR of $\mathbf{2 d}$

Page S12

${ }^{1} \mathrm{H}-{ }^{1} \mathrm{H}$ COSY of $\mathbf{2 d}$

Page S13

NOESY of $\mathbf{2 d}$

Page S14

${ }^{13} \mathrm{C}$ NMR of $\mathbf{2 d}$

Page S15

HMBC of $\mathbf{2 d}$

Page S16

${ }^{1} \mathrm{H}$ NMR of $\mathbf{3 e}$

Page S17

${ }^{1} \mathrm{H}-{ }^{1} \mathrm{H}$ COSY of $\mathbf{3 e}$

Page S18

ROESY of $\mathbf{3 e}$

Page S19

${ }^{13} \mathrm{C}$ NMR of $\mathbf{3 e}$

Page S20

HMBC of $3 \mathbf{e}$

Page S21-23

19,ap-21,23H-3 computational data

Page S24-26

19,sp-21,23H-3 computational data

Page S27-29

19,sp-22,24H-3 computational data

Page S30-32

19,ap-22,24H-3 computational data 

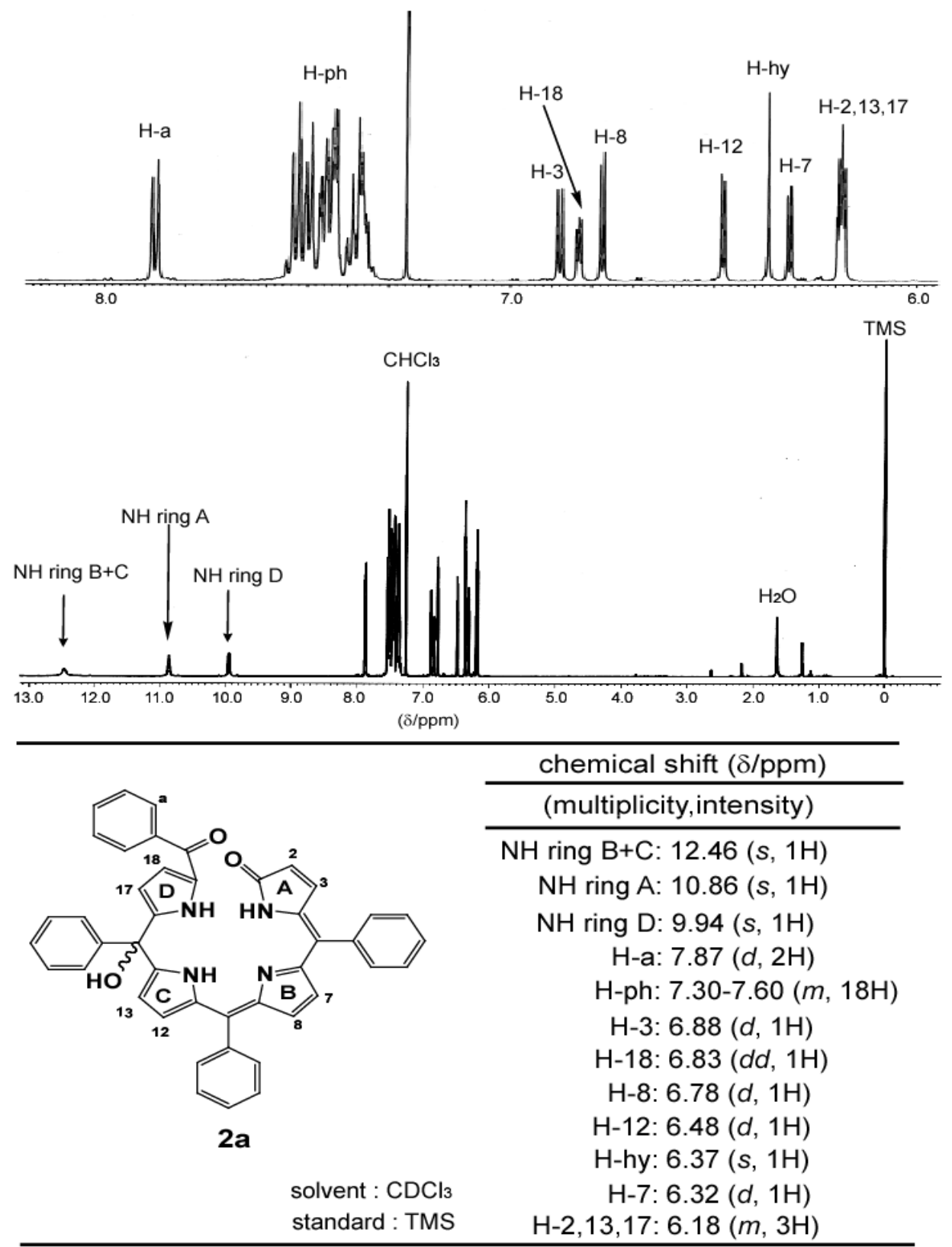

Figure S1. ${ }^{1} \mathrm{H}$ NMR spectrum of $\mathbf{2 a}$. 


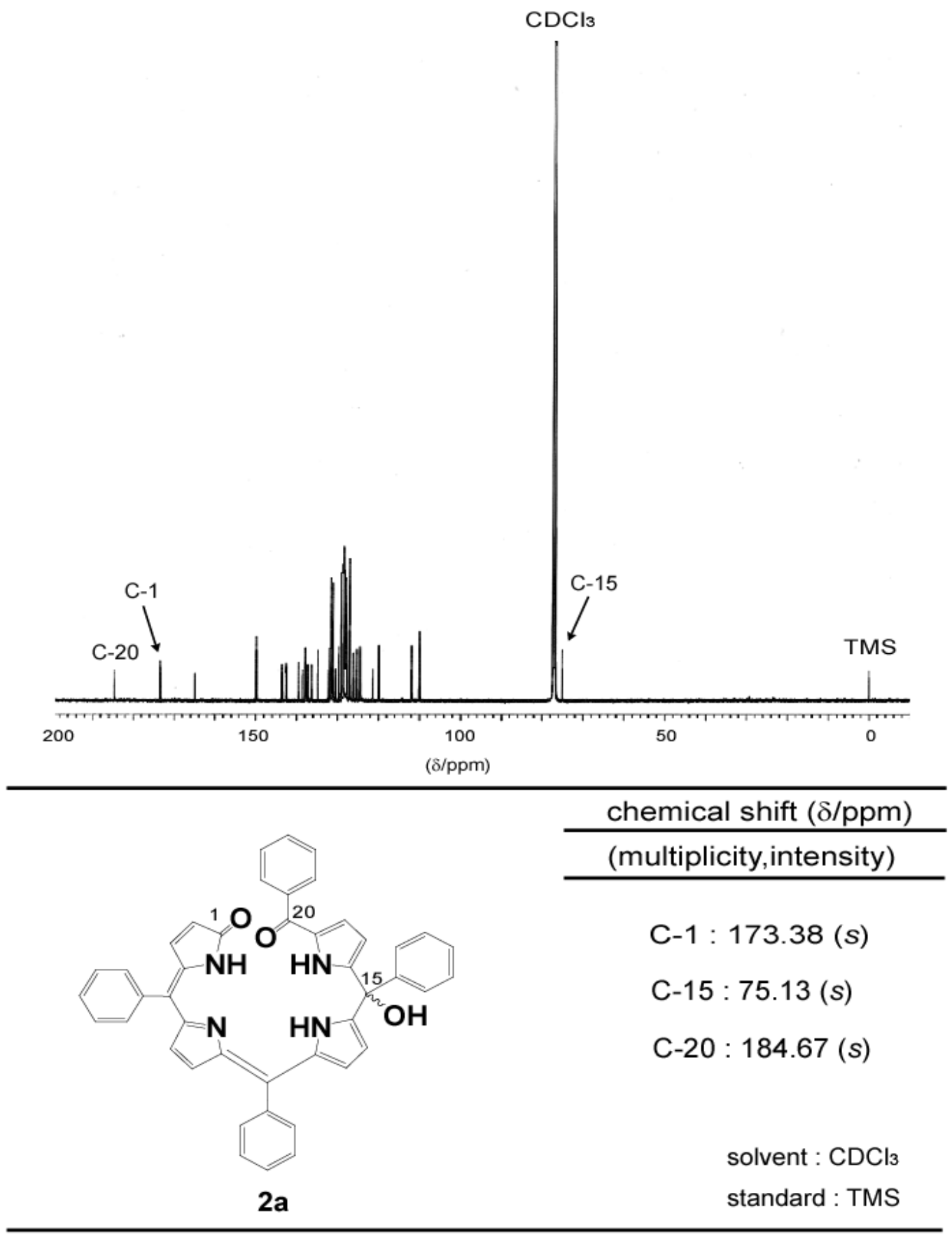

Figure S2. ${ }^{13} \mathrm{C}$ NMR spectrum of $\mathbf{2 a}$. 


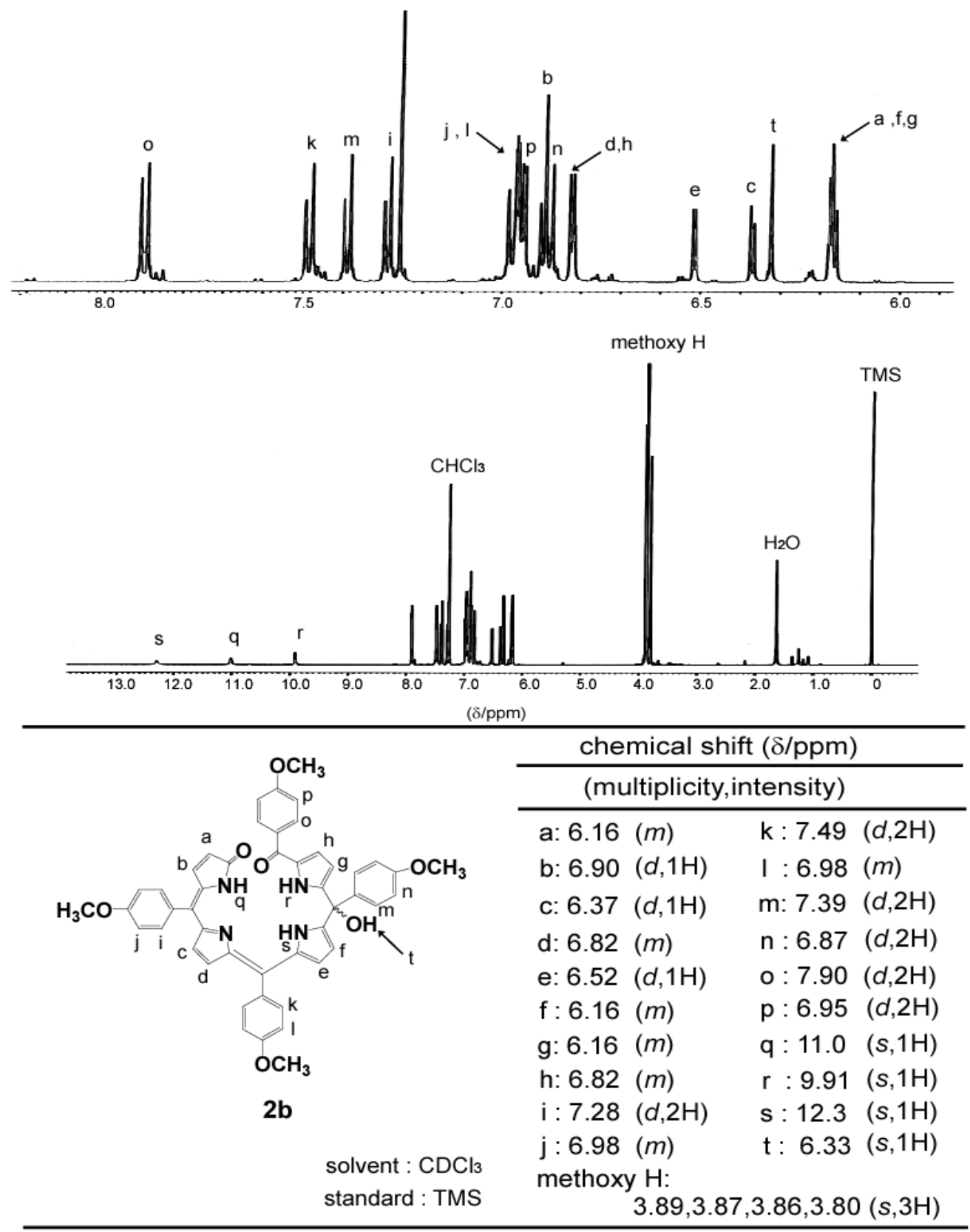

Figure S3. ${ }^{1} \mathrm{H}$ NMR spectrum of $\mathbf{2 b}$. 


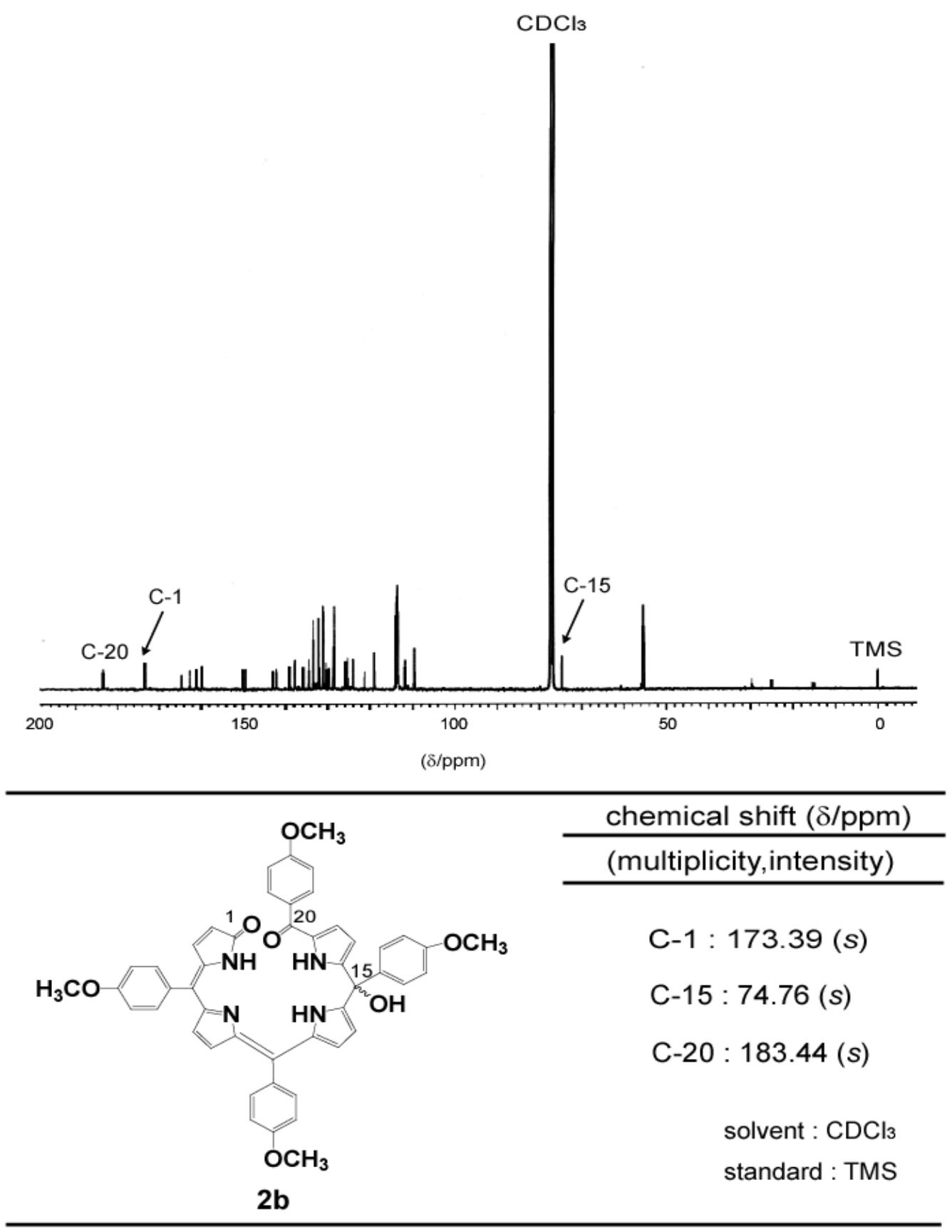

Figure S4. ${ }^{13} \mathrm{C}$ NMR spectrum of $\mathbf{2 b}$. 

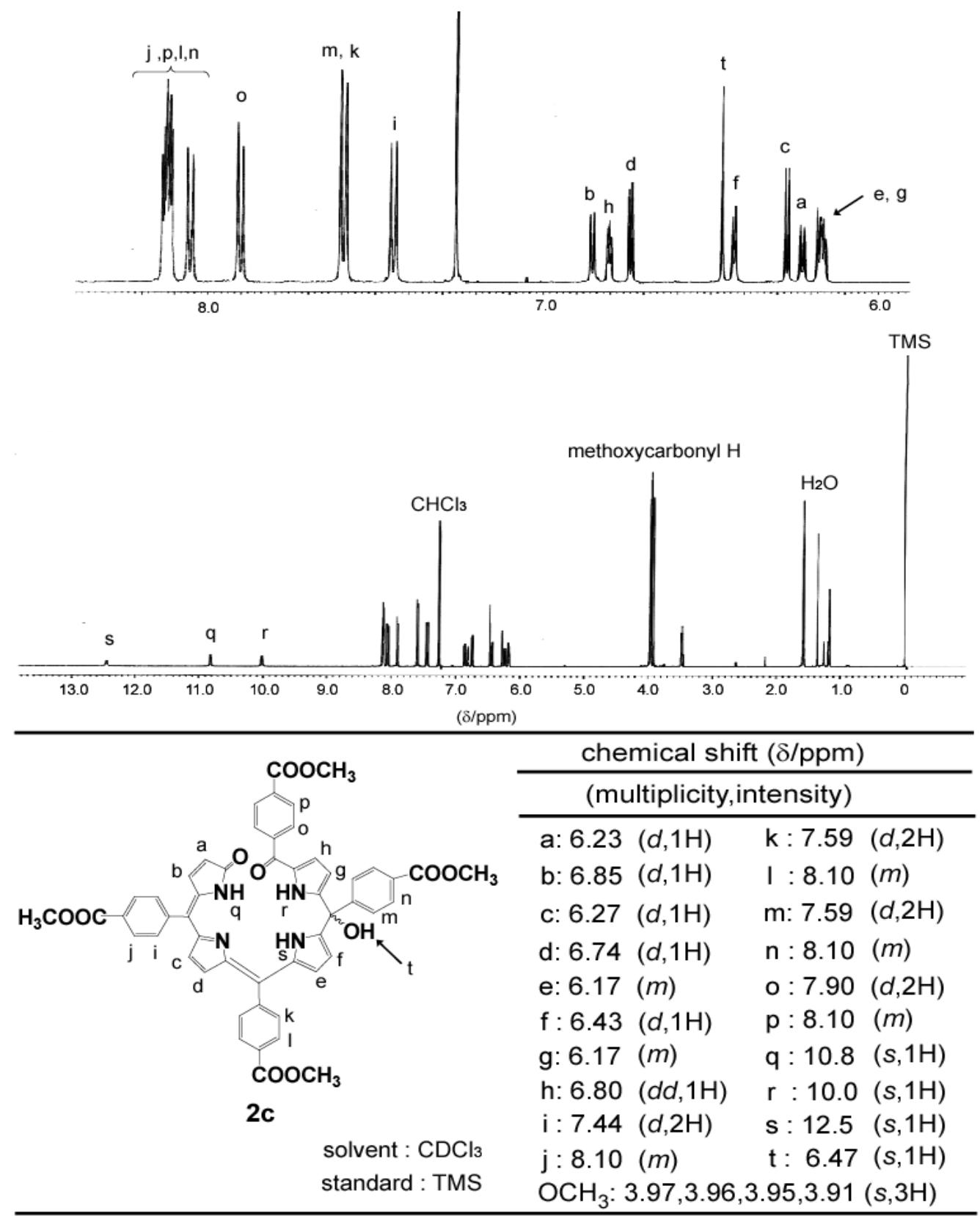

Figure S5. ${ }^{1} \mathrm{H}$ NMR spectrum of $2 \mathbf{c}$. 


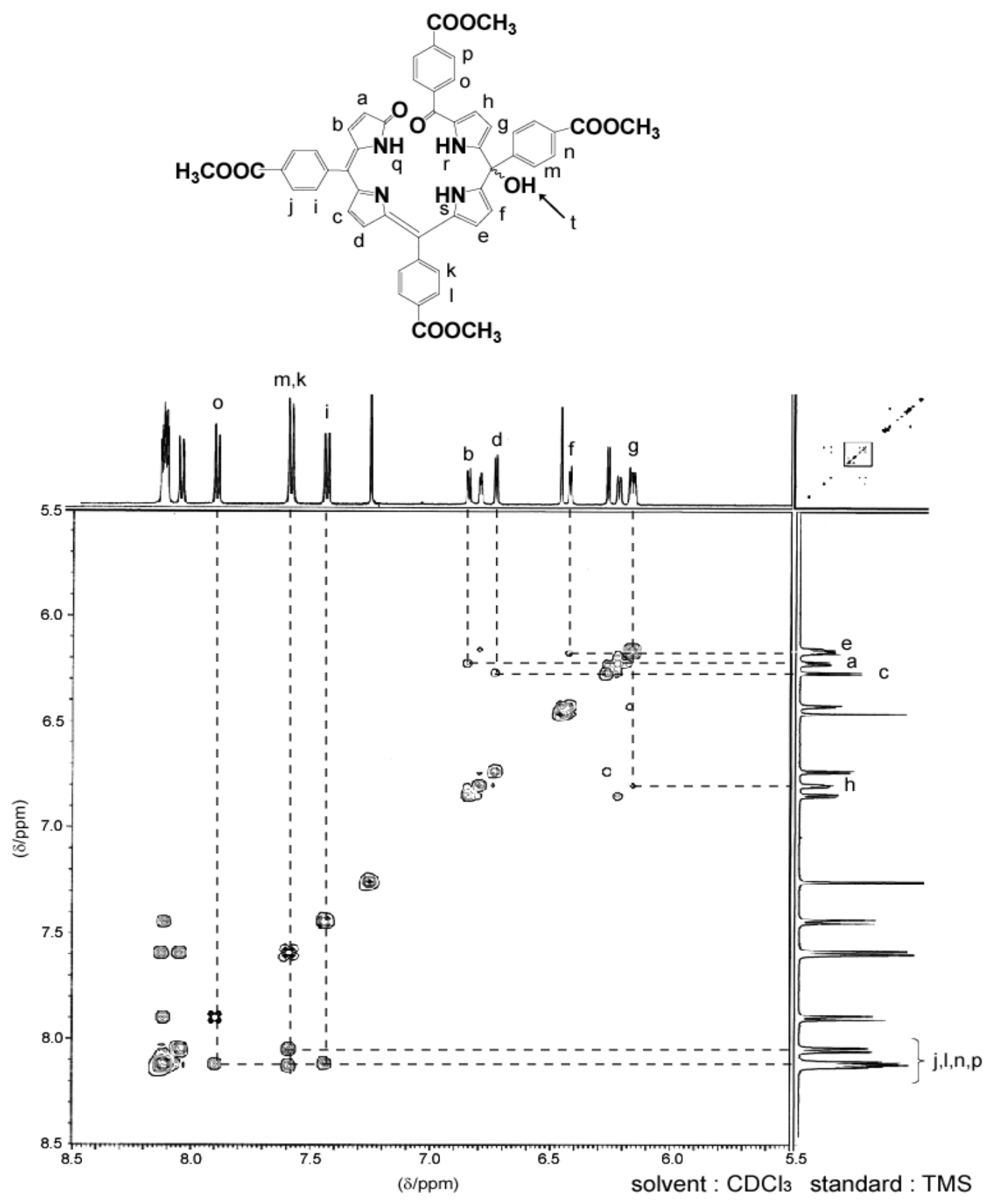

Figure S6. COSY spectrum of $2 \mathbf{c}$. 


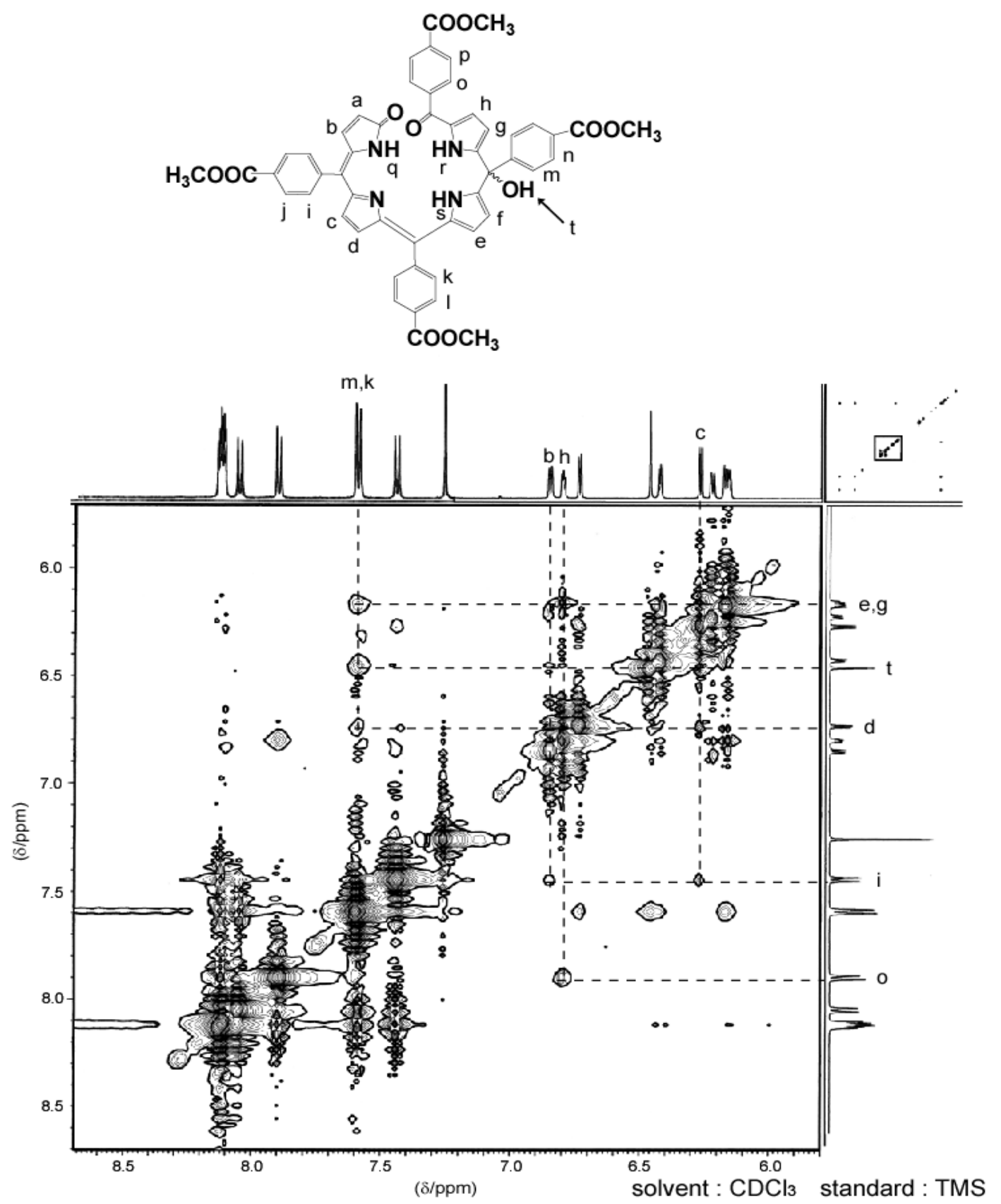

Figure S7. NOESY spectrum of $2 \mathbf{c}$. 


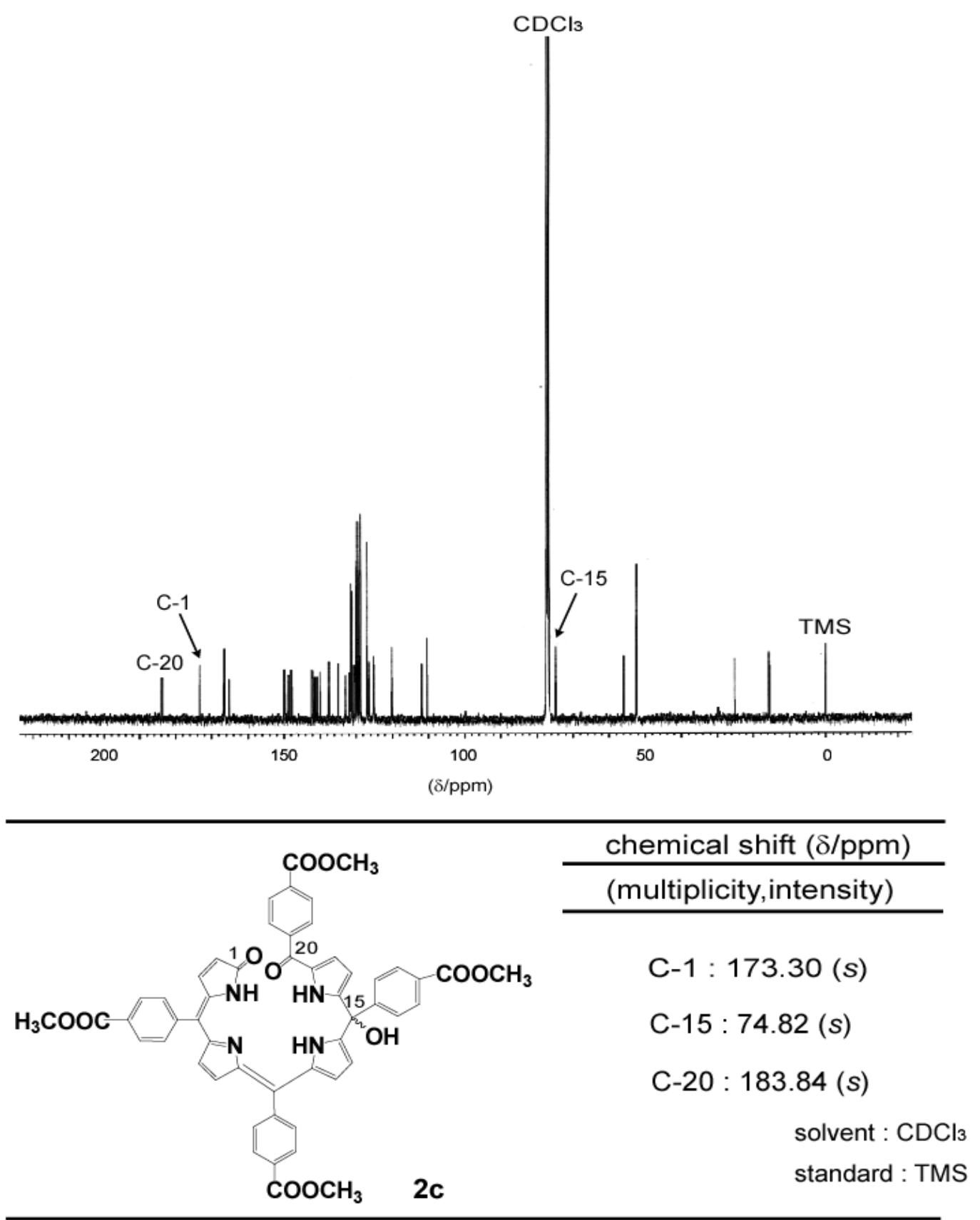

Figure S8. ${ }^{13} \mathrm{C}$ NMR spectrum of $2 \mathrm{c}$. 


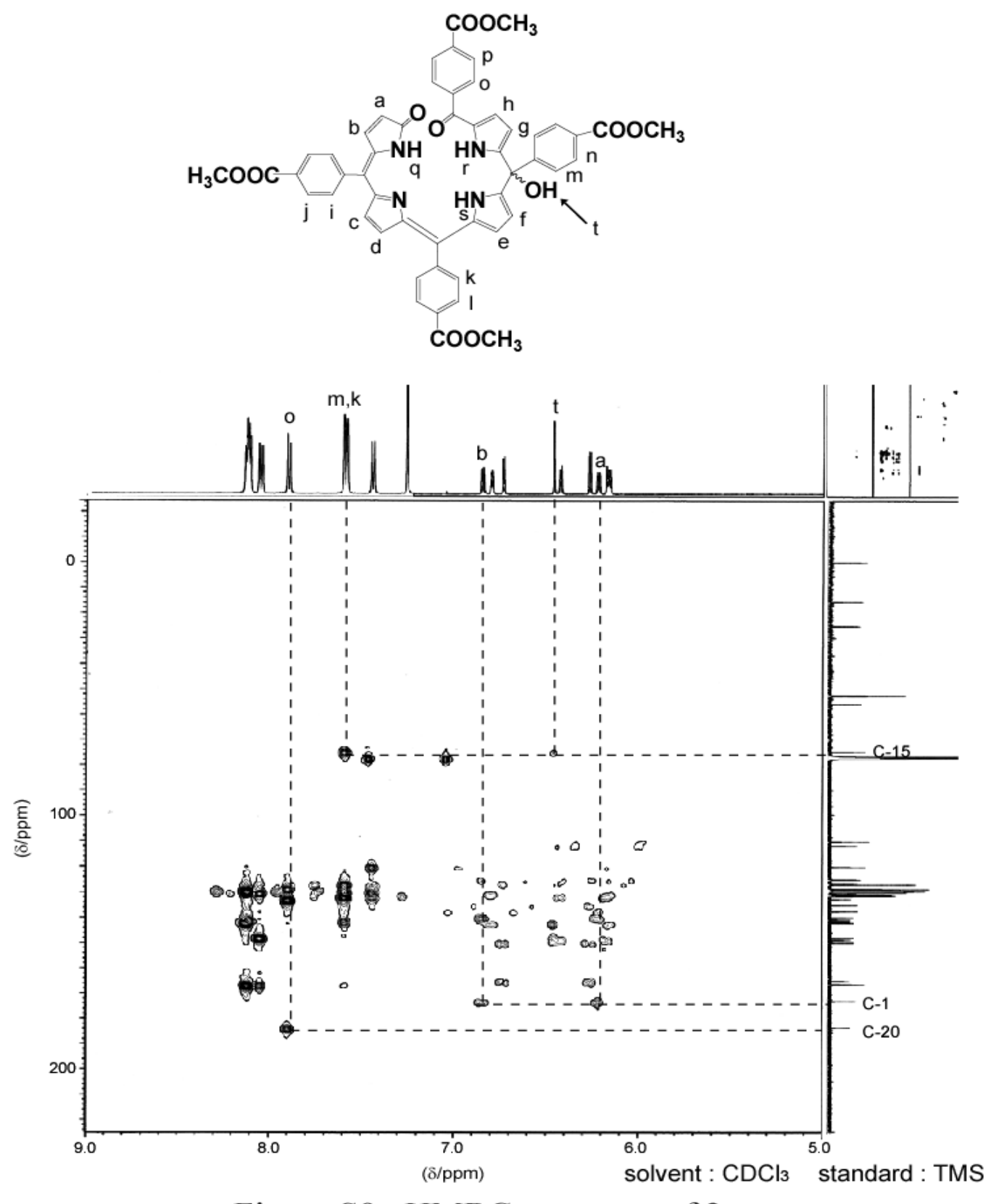

Figure S9. HMBC spectrum of $2 \mathbf{c}$. 

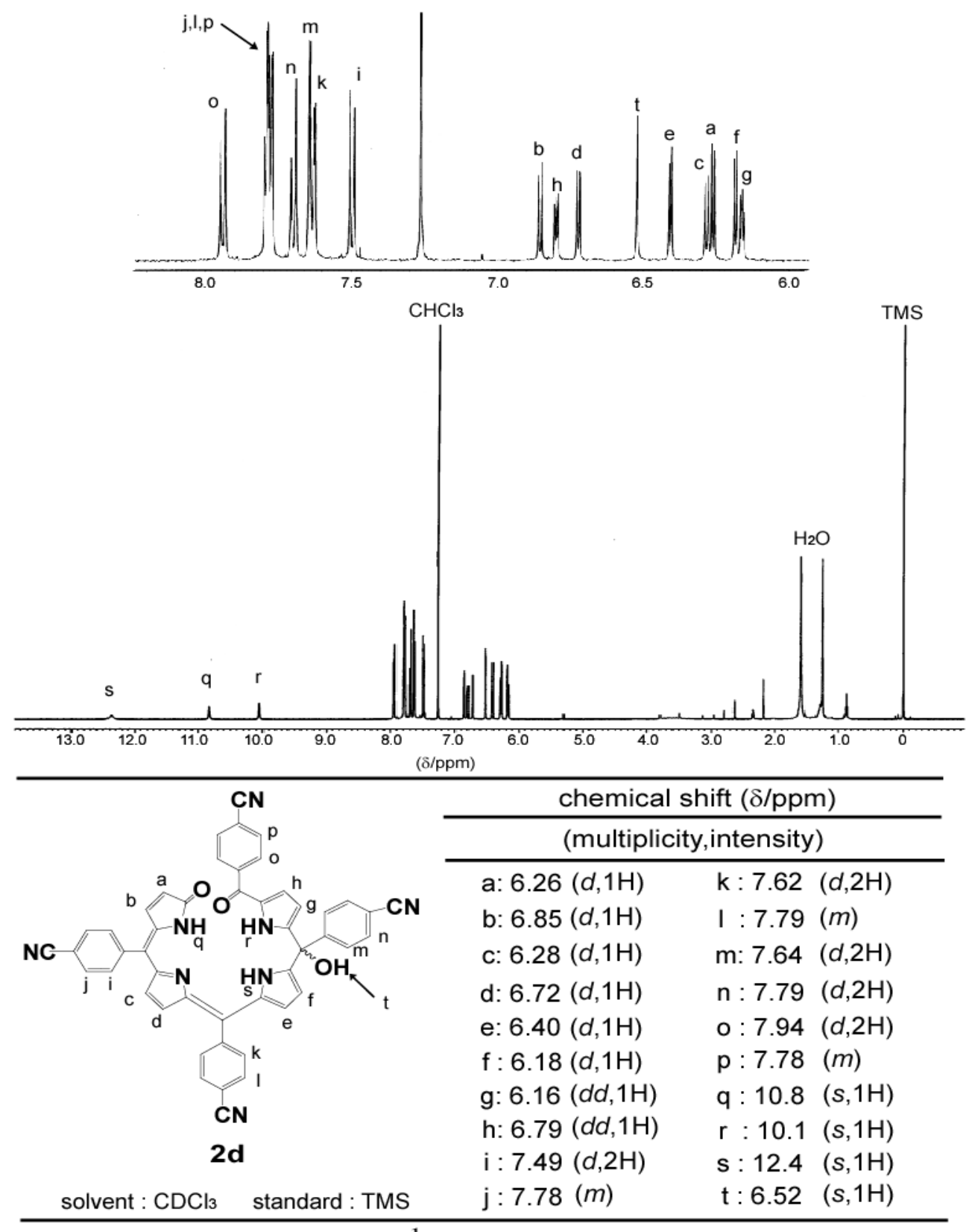

Figure S10. ${ }^{1} \mathrm{H}$ NMR spectrum of $\mathbf{2 d}$. 


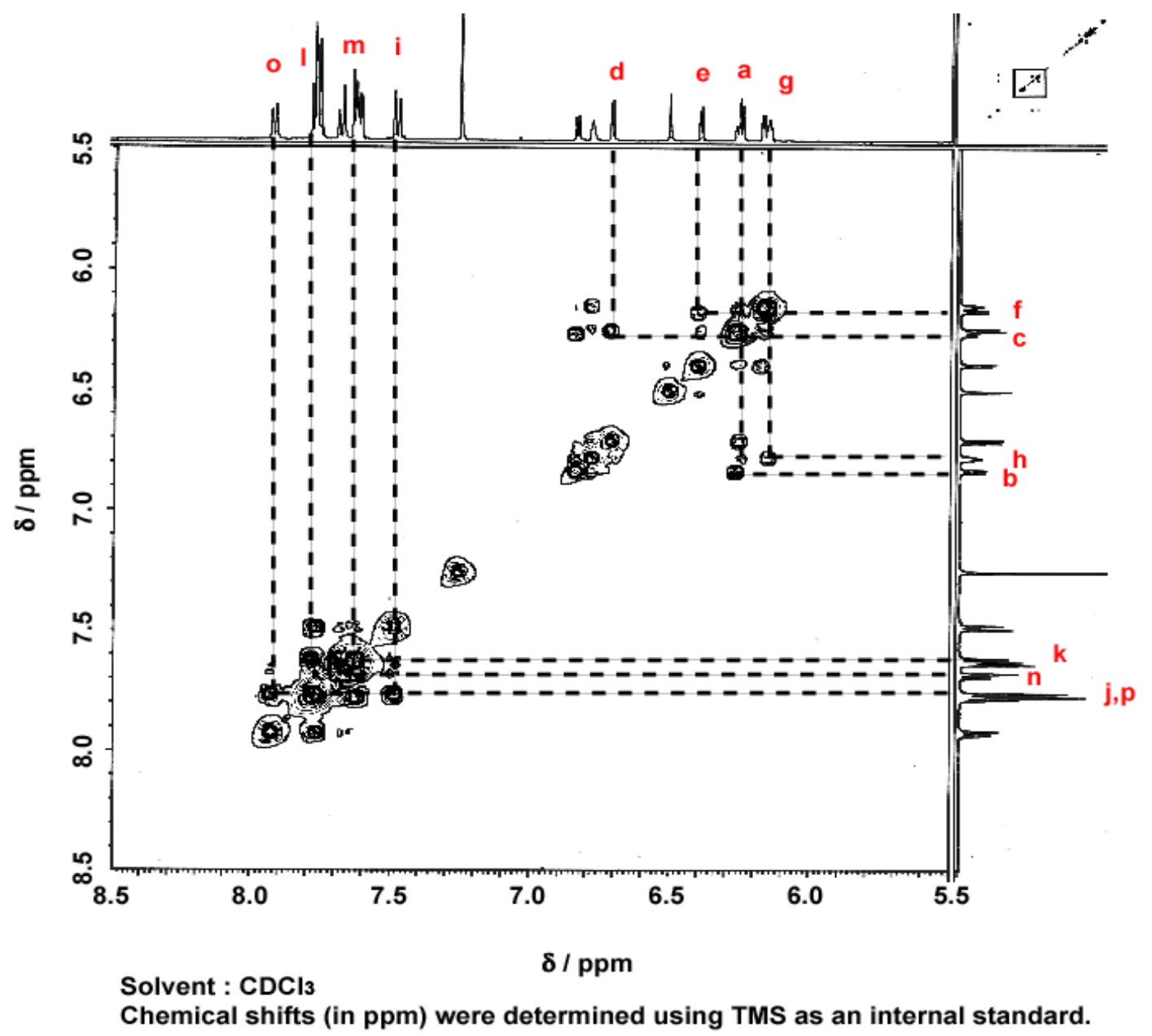

Figure $\mathrm{S} 11 .{ }^{1} \mathrm{H}-{ }^{1} \mathrm{H}$ COSY spectrum of $\mathbf{2 d}$. 


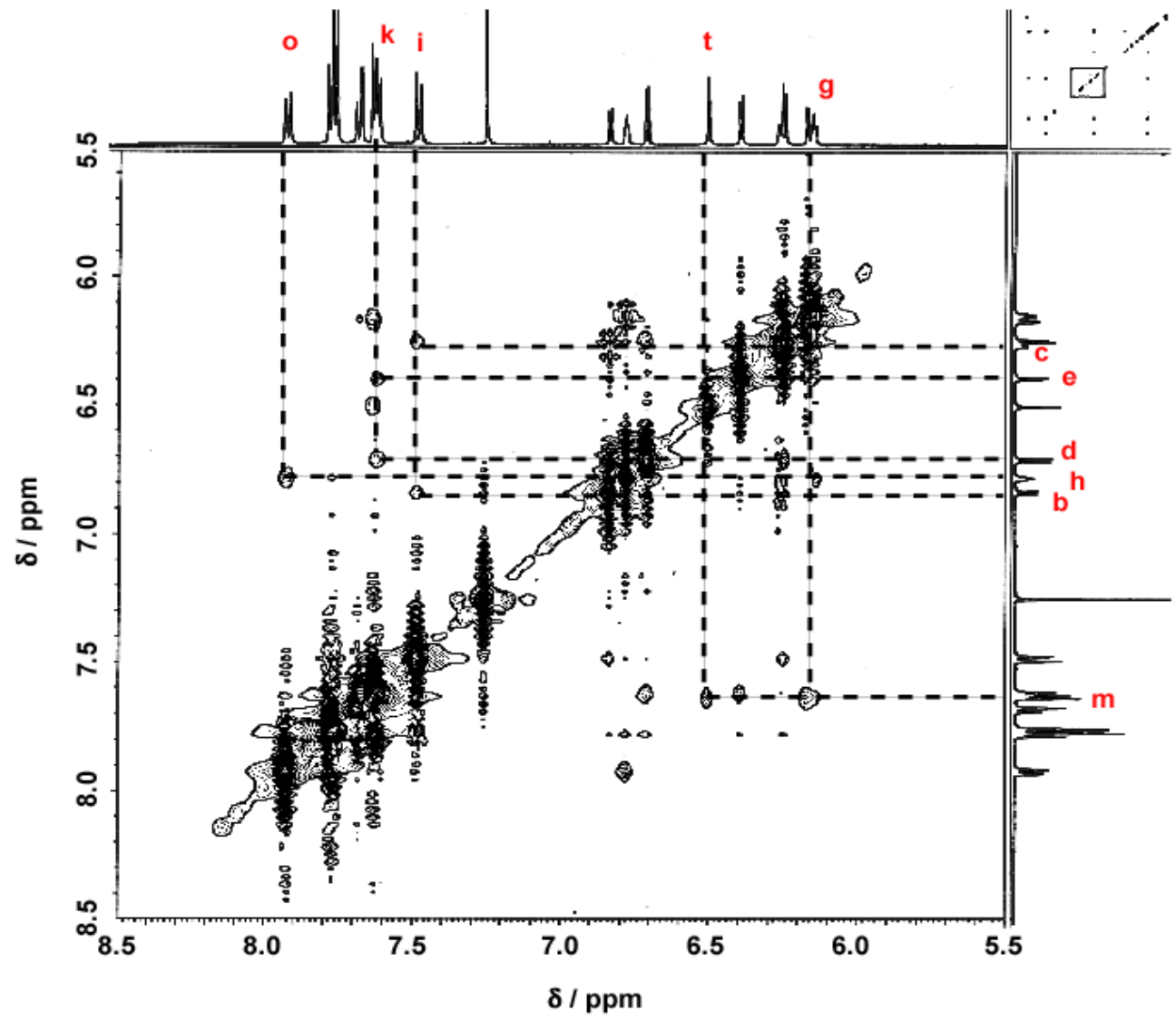

Solvent : $\mathrm{CDCl}_{3}$

Chemical shifts (in ppm) were determined using TMS as an internal standard.

Figure S12. NOESY spectrum of $\mathbf{2 d}$. 


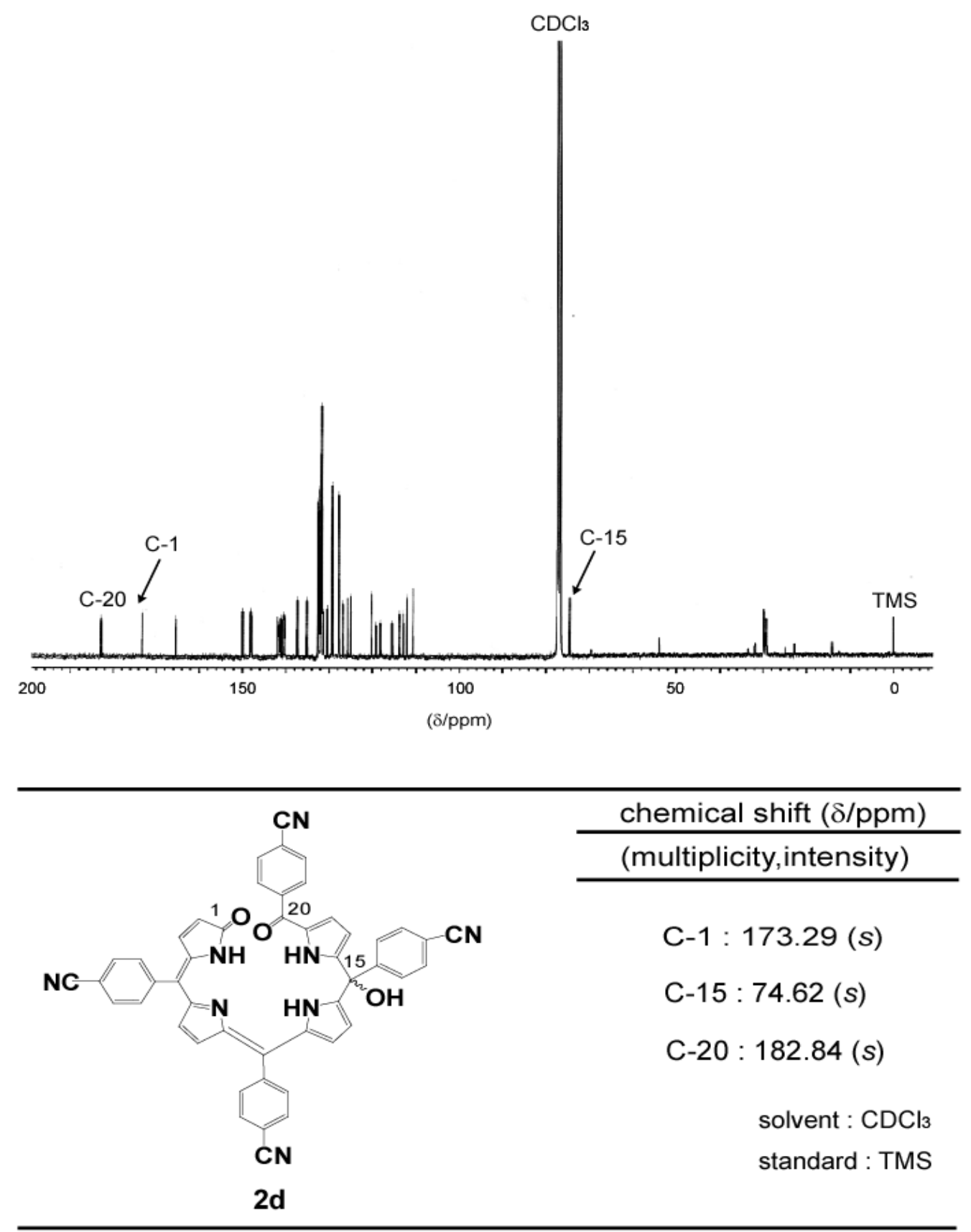

Figure S13. ${ }^{13} \mathrm{C}$ NMR spectrum of $\mathbf{2 d}$. 


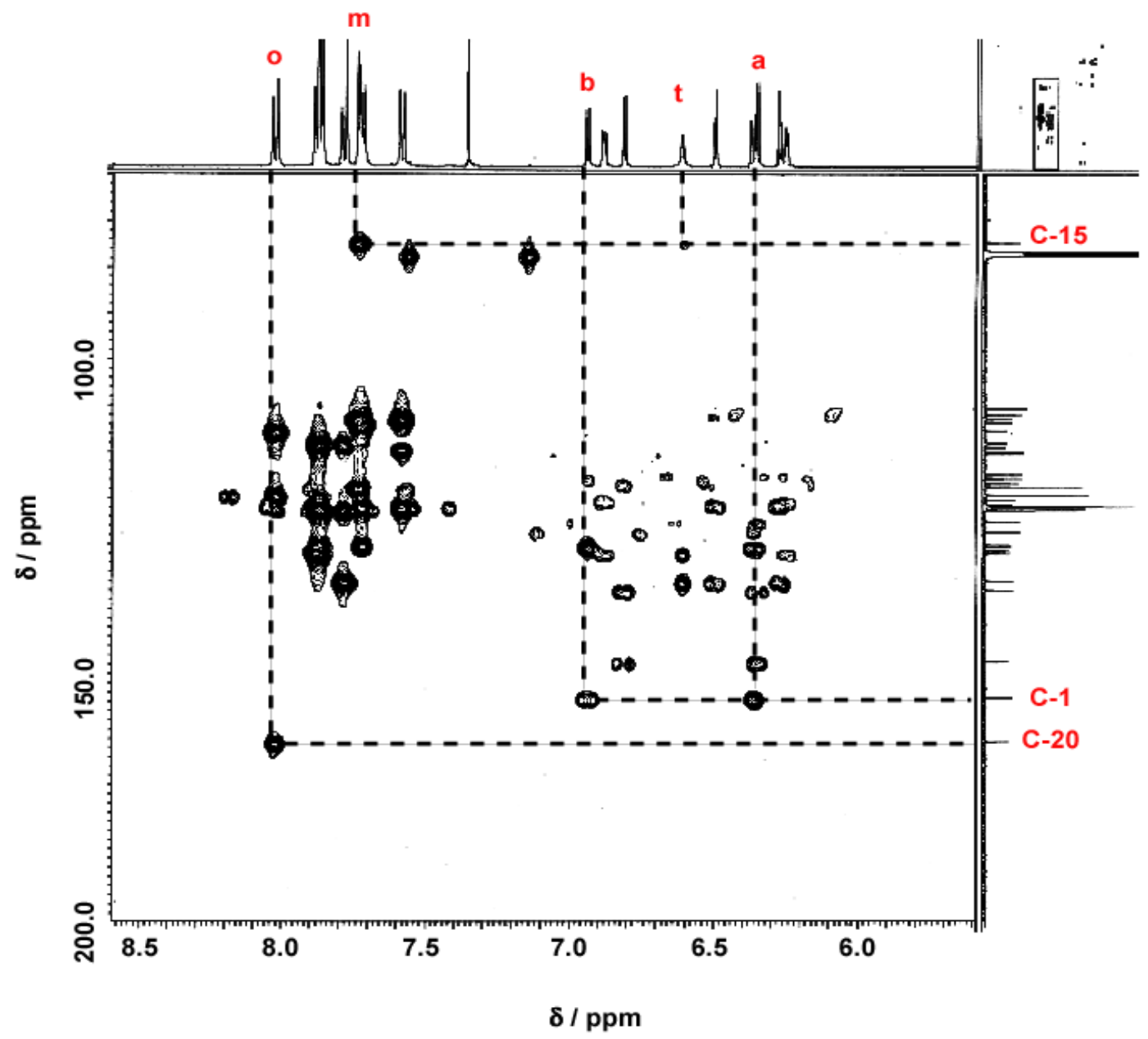

Solvent : $\mathrm{CDCl}_{3}$

Chemical shifts (in ppm) were determined using TMS as an internal standard.

Figure S14. HMBC spectrum of $\mathbf{2 d}$. 


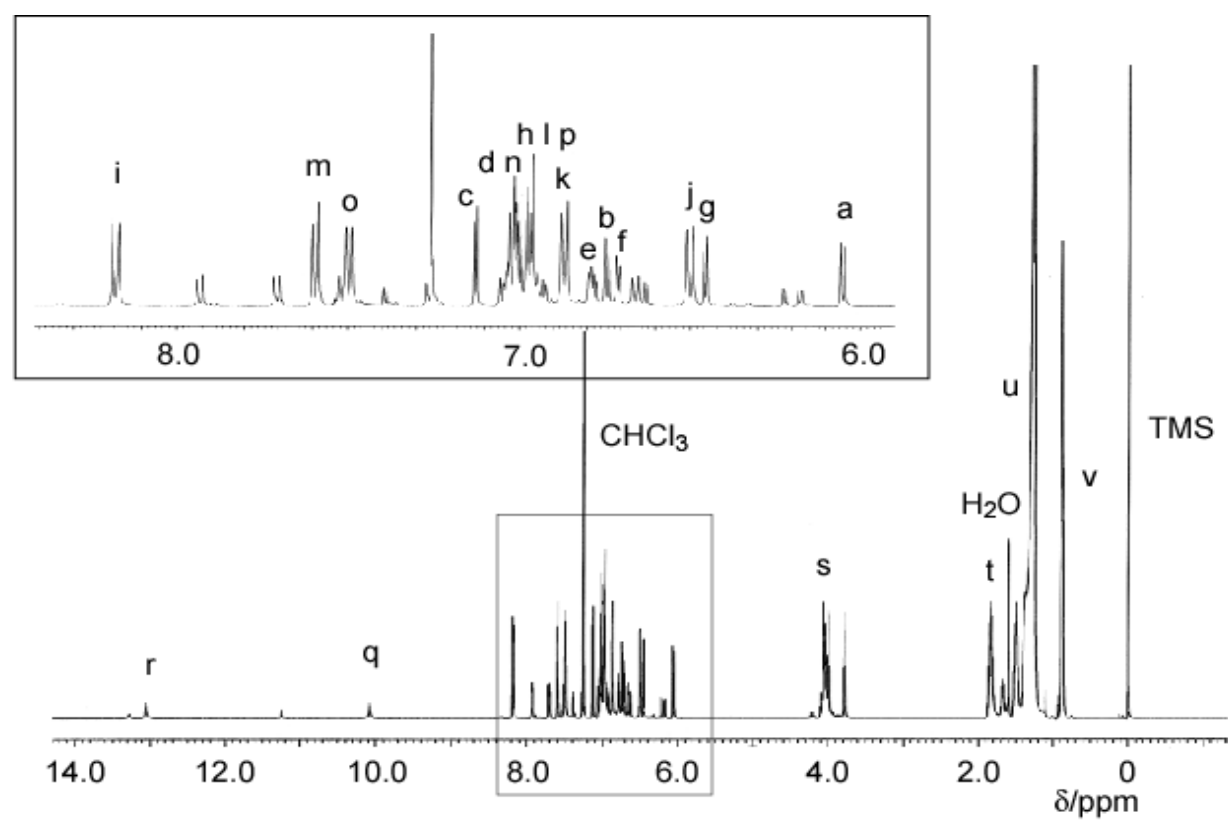

chemical shift $(\delta / p p m)$

(multiplicity, intensity)

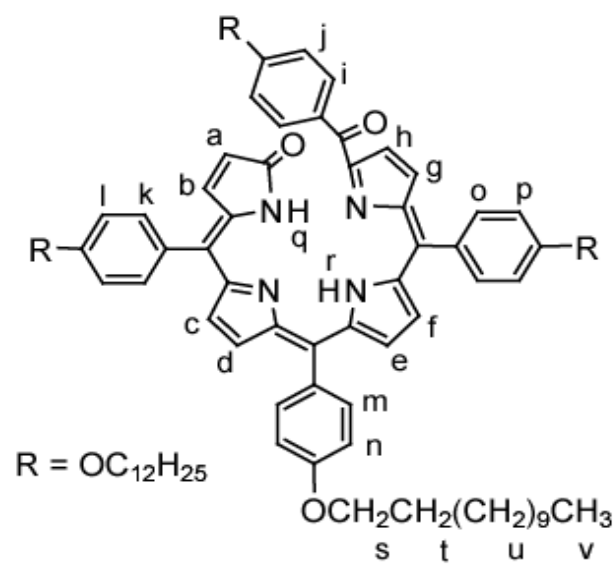

r : $13.05(b r s, 1 \mathrm{H})$

b : $6.74(d, 1 \mathrm{H})$

$\mathrm{q}: 10.08($ br s, $1 \mathrm{H})$

f : $6.72(d, 1 \mathrm{H})$

i : $8.18(d, 2 \mathrm{H})$

j : $6.50(d, 2 \mathrm{H})$

$\mathrm{m}: 7.59(d, 2 \mathrm{H})$

$\mathrm{g}: 6.45(d, 1 \mathrm{H})$

$0: 7.50(d, 2 \mathrm{H})$

a : $6.05(d, 1 \mathrm{H})$

c : $7.13(d, 1 \mathrm{H})$

s : $3.96 \sim 4.09(m, 8 \mathrm{H})$

$\mathrm{d} \mathrm{n}: 6.99 \sim 7.03(m, 3 \mathrm{H}) \quad \mathrm{t}: 1.73 \sim 1.89(\mathrm{~m}, 8 \mathrm{H})$

h I p : $6.95 \sim 6.99(m, 5 \mathrm{H})$ u : $1.26 \sim 1.49(m, 72 \mathrm{H})$

$\mathrm{k}: 6.87(d, 2 \mathrm{H})$

v: $0.88(t, 12 \mathrm{H})$

$\mathrm{e}: 6.79(d, 1 \mathrm{H})$

solvent : $\mathrm{CDCl}_{3}$ standard: TMS

temperature : $25^{\circ} \mathrm{C}$

Figure S15. ${ }^{1} \mathrm{H}$ NMR spectrum of $3 e$. 

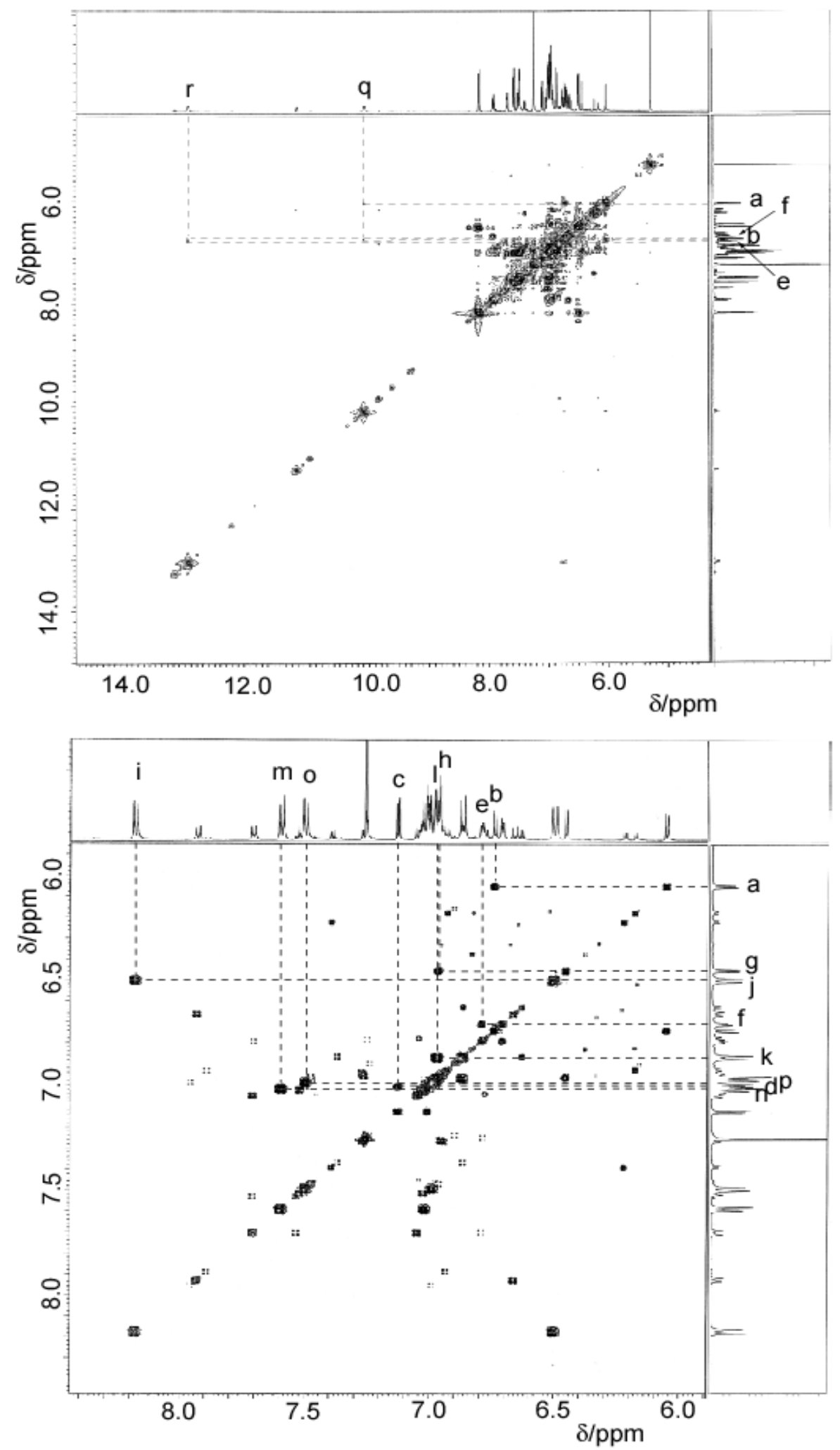

Figure S16. HH COSY spectrum of $3 e$ in $\mathrm{CDCl}_{3}$. 


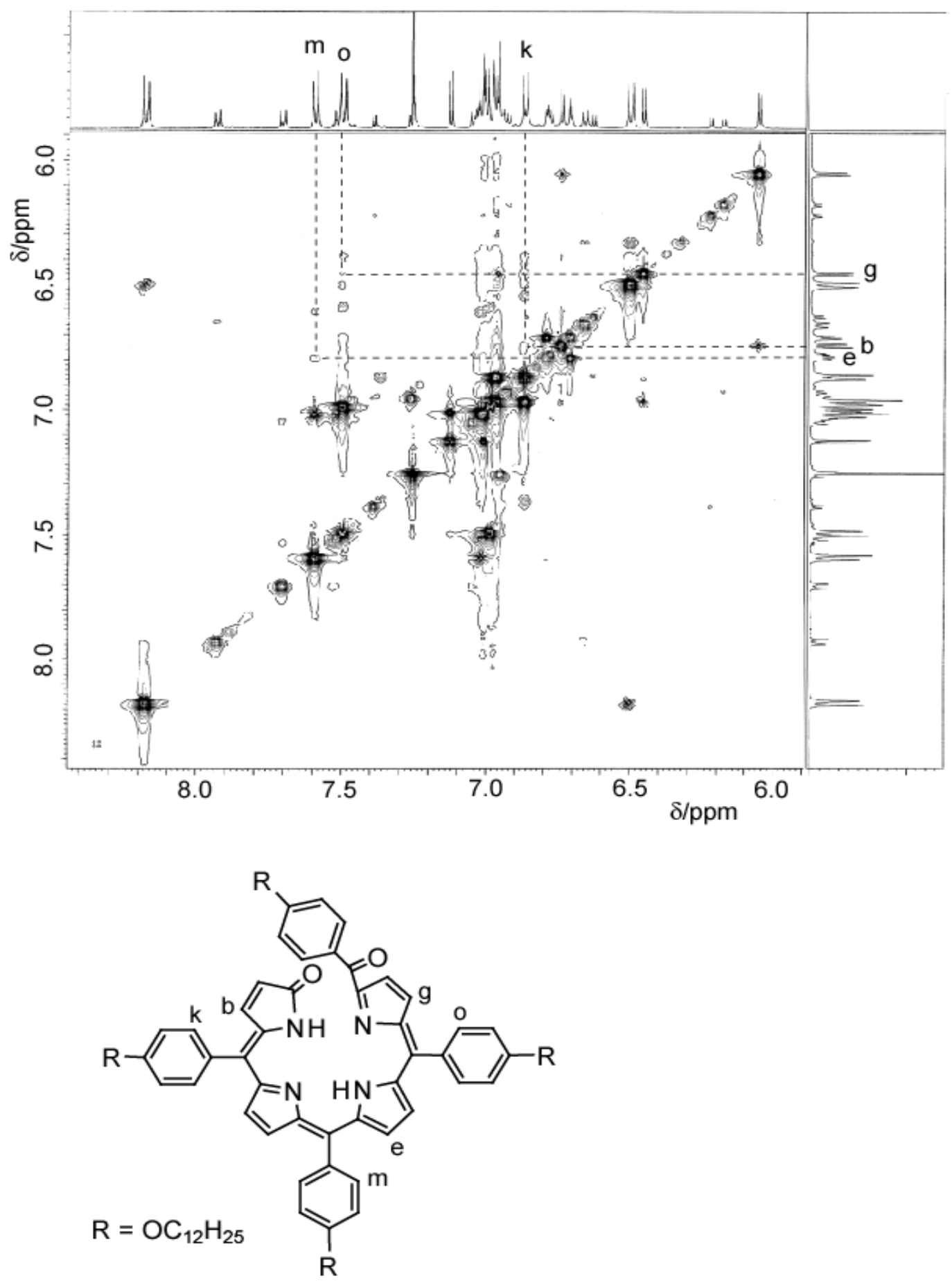

Figure S17. ROESY spectrum of $3 e$ in $\mathrm{CDCl}_{3}$. 


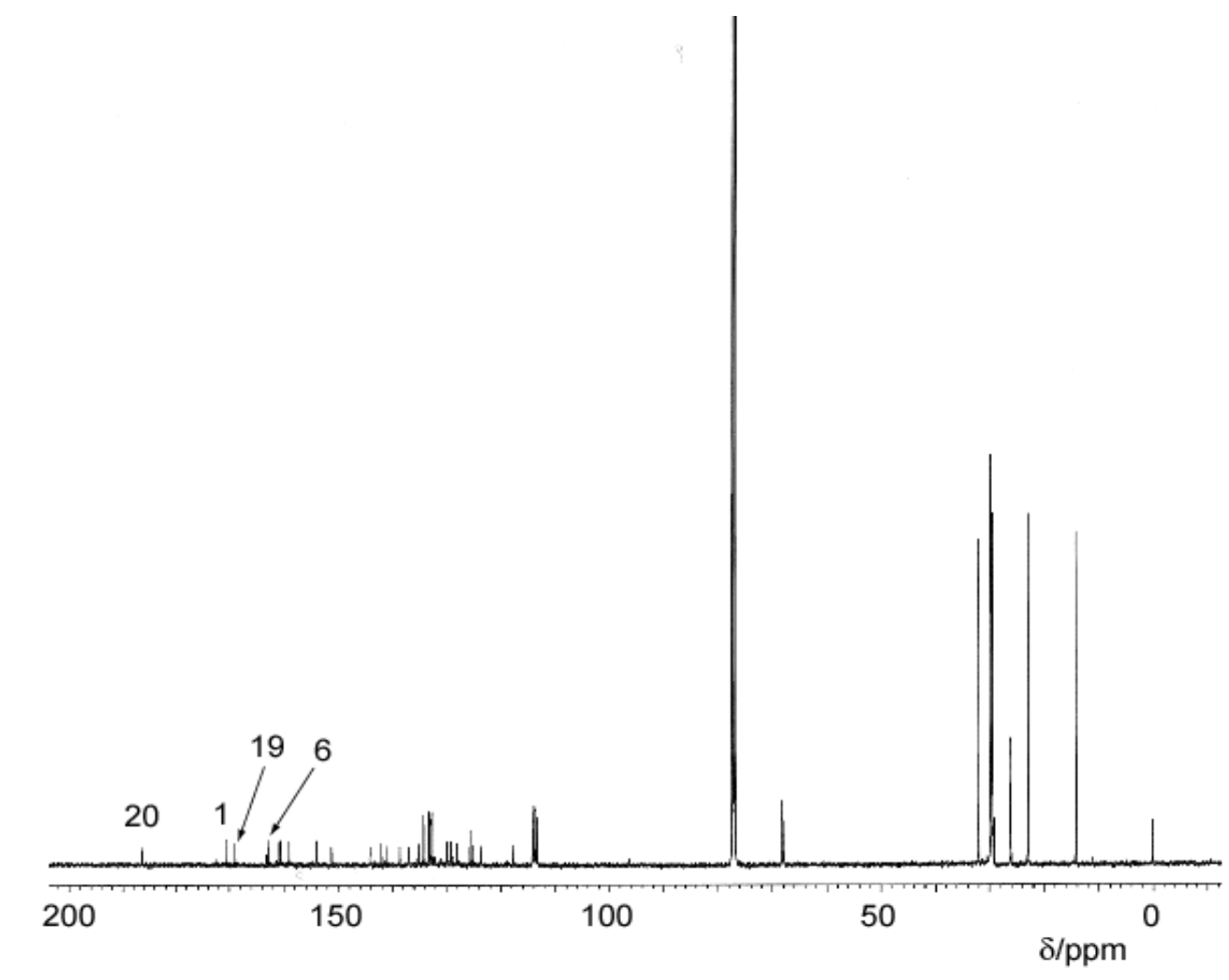

chemical shift $(\delta / p p m)$

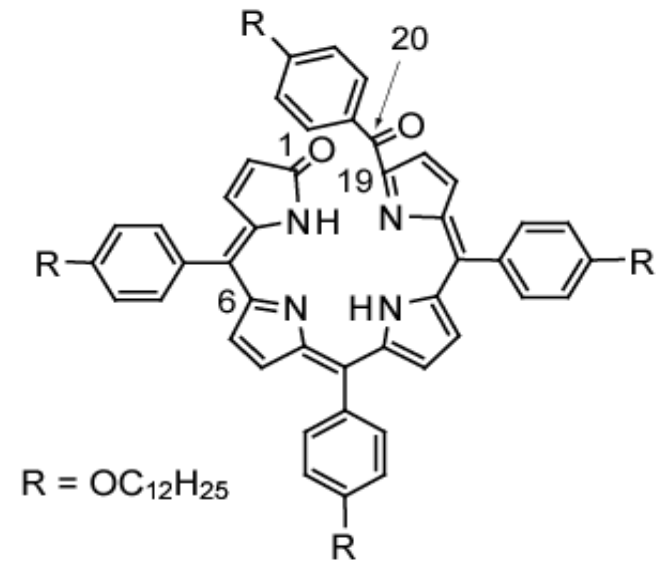

20 : 186.3 ( benzoyl )

$1: 170.5$ (lactam )

19 : 169.1 (imide )

$6: 162.8$ ( imide )

solvent : $\mathrm{CDCl}_{3}$

standard: TMS

temperature : $25^{\circ} \mathrm{C}$

Figure S18. ${ }^{13} \mathrm{C}$ NMR spectrum of $3 e$. 

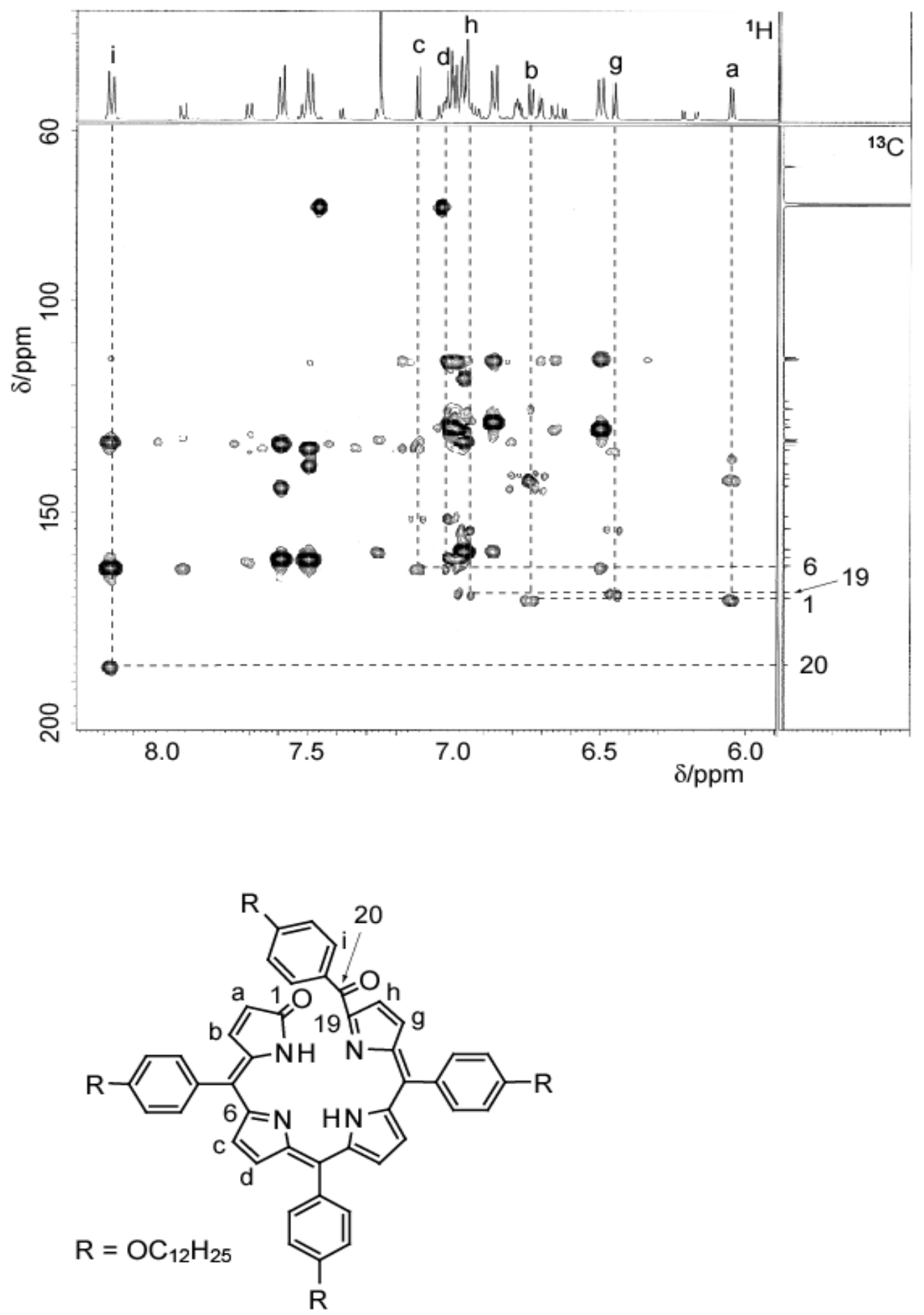

Figure S19. HMBC spectrum of $3 e$ in $\mathrm{CDCl}_{3}$. 
19,ap-21,23H-3 computational data (B3LYP/6-31G*)

Energy: -2064.18357965 a.u.

\begin{tabular}{|c|c|c|c|}
\hline \multirow{2}{*}{$\begin{array}{l}\text { Atomic } \\
\text { Number }\end{array}$} & \multicolumn{3}{|c|}{ Coordinates (Angstroms) } \\
\hline & $\mathrm{X}$ & $\mathrm{Y}$ & $\mathrm{Z}$ \\
\hline 6 & 2.807010 & -3.305163 & 0.620996 \\
\hline 6 & 1.667115 & -3.864961 & 1.112067 \\
\hline 6 & 0.763797 & -2.760495 & 1.381133 \\
\hline 7 & 1.291008 & -1.588556 & 1.058596 \\
\hline 6 & 2.571651 & -1.869041 & 0.581349 \\
\hline 6 & 3.432159 & -0.874544 & 0.128255 \\
\hline 6 & 3.031470 & 0.495060 & -0.038371 \\
\hline 7 & 1.730163 & 0.926332 & 0.016395 \\
\hline 6 & 1.654421 & 2.280038 & -0.166881 \\
\hline 6 & 2.983395 & 2.742337 & -0.337998 \\
\hline 6 & 3.829631 & 1.641845 & -0.271223 \\
\hline 6 & 0.424600 & 3.029675 & -0.213272 \\
\hline 6 & -0.813538 & 2.429877 & -0.424079 \\
\hline 7 & -0.960169 & 1.093638 & -0.763858 \\
\hline 6 & -2.273263 & 0.867329 & -0.878449 \\
\hline 6 & -3.037913 & 2.080843 & -0.589894 \\
\hline 6 & -2.128420 & 3.052366 & -0.313931 \\
\hline 6 & -2.832097 & -0.405117 & -1.293121 \\
\hline 6 & -2.026663 & -1.429587 & -1.749948 \\
\hline 6 & -2.405639 & -2.803067 & -2.105556 \\
\hline 6 & -1.294778 & -3.482919 & -2.457431 \\
\hline 6 & -0.122636 & -2.569457 & -2.352611 \\
\hline 8 & 1.057433 & -2.780034 & -2.578547 \\
\hline 7 & -0.664339 & -1.353851 & -1.926393 \\
\hline 6 & -4.309582 & -0.594708 & -1.245093 \\
\hline 6 & -5.016516 & -0.427186 & -0.041523 \\
\hline 6 & -6.398649 & -0.605968 & 0.003400 \\
\hline 6 & -7.102680 & -0.955850 & -1.151122 \\
\hline 6 & -6.414007 & -1.122497 & -2.353263 \\
\hline 6 & -5.031417 & -0.938976 & -2.400492 \\
\hline
\end{tabular}




\begin{tabular}{|c|c|c|c|}
\hline & 0.523929 & 4.503016 & -0.049947 \\
\hline & 1.243425 & 5.061462 & 1.022508 \\
\hline & 1.327312 & 6.443227 & 1.182125 \\
\hline & 0.705385 & 7.295773 & 0.267092 \\
\hline & -0.002130 & 6.756100 & -0.808562 \\
\hline & -0.093161 & 5.373798 & -0.966174 \\
\hline & 4.840245 & -1.215537 & -0.205913 \\
\hline & 5.655887 & -1.875668 & 0.729120 \\
\hline & 6.976790 & -2.195960 & 0.418186 \\
\hline & 7.501536 & -1.871292 & -0.833986 \\
\hline & 6.698972 & -1.220293 & -1.773431 \\
\hline & 5.381806 & -0.888273 & -1.461328 \\
\hline & -0.608376 & -2.969132 & 1.923971 \\
\hline & -1.089437 & -4.097624 & 1.833952 \\
\hline & -1.387814 & -1.870626 & 2.582512 \\
\hline & -2.741815 & -2.128626 & 2.856697 \\
\hline 6 & -3.525832 & -1.183259 & 3.510724 \\
\hline 6 & -2.964928 & 0.034933 & 3.908716 \\
\hline 6 & -1.618782 & 0.296614 & 3.649706 \\
\hline 6 & -0.830930 & -0.648108 & 2.991375 \\
\hline 1 & 3.700075 & -3.809107 & 0.279549 \\
\hline 1 & 1.438228 & -4.908413 & 1.275304 \\
\hline 1 & 0.948904 & 0.307754 & 0.227092 \\
\hline 1 & 3.263660 & 3.769029 & -0.522297 \\
\hline 1 & 4.905573 & 1.642181 & -0.364136 \\
\hline 1 & -4.115136 & 2.169512 & -0.609787 \\
\hline 1 & -2.323388 & 4.078265 & -0.034562 \\
\hline 1 & -3.419795 & -3.173220 & -2.050865 \\
\hline 1 & -1.205611 & -4.517648 & -2.758724 \\
\hline 1 & -0.148156 & -0.514887 & -1.679777 \\
\hline & -4.472030 & -0.177187 & 0.864290 \\
\hline 1 & -6.926329 & -0.479913 & 0.945325 \\
\hline 1 & -8.179744 & -1.095473 & -1.113845 \\
\hline 1 & -6.953031 & -1.386396 & -3.259417 \\
\hline & -4.502143 & -1.046132 & -3.343270 \\
\hline & 1.720724 & 4.402936 & 1.741893 \\
\hline
\end{tabular}




$\begin{array}{lrrr}1 & 1.876617 & 6.854633 & 2.024666 \\ 1 & 0.775545 & 8.373156 & 0.389561 \\ 1 & -0.478149 & 7.411798 & -1.532639 \\ 1 & -0.625676 & 4.957326 & -1.815517 \\ 1 & 5.251388 & -2.118809 & 1.706838 \\ 1 & 7.596494 & -2.697339 & 1.156636 \\ 1 & 8.529724 & -2.125097 & -1.077239 \\ 1 & 7.096730 & -0.974971 & -2.754359 \\ 1 & 4.754929 & -0.398141 & -2.199741 \\ 1 & -3.153733 & -3.084700 & 2.551464 \\ 1 & -4.571338 & -1.396337 & 3.718495 \\ 1 & -3.574227 & 0.773198 & 4.424089 \\ 1 & -1.177903 & 1.238827 & 3.964081 \\ 1 & 0.213374 & -0.444410 & 2.792476\end{array}$


19,sp-21,23H-3 computational data (B3LYP/6-31G*)

Energy: -2064.17988904 a.u.

\begin{tabular}{|c|c|c|c|}
\hline \multirow{2}{*}{$\begin{array}{l}\text { Atomic } \\
\text { Number }\end{array}$} & \multicolumn{3}{|c|}{ Coordinates (Angstroms) } \\
\hline & $X$ & $\mathrm{Y}$ & $\mathrm{Z}$ \\
\hline 6 & -0.843304 & -3.881740 & -0.010194 \\
\hline 6 & 0.474447 & -3.805779 & -0.361101 \\
\hline 6 & 0.709323 & -2.424776 & -0.749568 \\
\hline 7 & -0.389524 & -1.690614 & -0.639495 \\
\hline 6 & -1.386370 & -2.548236 & -0.199238 \\
\hline 6 & -2.689617 & -2.104921 & 0.040582 \\
\hline 6 & -3.064822 & -0.725527 & -0.036911 \\
\hline 7 & -2.166633 & 0.299401 & -0.201894 \\
\hline 6 & -2.805905 & 1.503188 & -0.268852 \\
\hline 6 & -4.195686 & 1.237267 & -0.146153 \\
\hline 6 & -4.354180 & -0.132918 & 0.007325 \\
\hline 6 & -2.133571 & 2.769828 & -0.416274 \\
\hline 6 & -0.781659 & 2.927054 & -0.124129 \\
\hline 7 & -0.031145 & 1.955980 & 0.515173 \\
\hline 6 & 1.224434 & 2.409899 & 0.562313 \\
\hline 6 & 1.328597 & 3.726674 & -0.071319 \\
\hline 6 & 0.075930 & 4.055557 & -0.480712 \\
\hline 6 & 2.306365 & 1.701201 & 1.207859 \\
\hline 6 & 2.081697 & 0.567979 & 1.960124 \\
\hline 6 & 3.069904 & -0.308456 & 2.598234 \\
\hline 6 & 2.422496 & -1.343346 & 3.172766 \\
\hline 6 & 0.959569 & -1.181457 & 2.934440 \\
\hline 8 & 0.032610 & -1.904110 & 3.262286 \\
\hline 7 & 0.850099 & 0.016815 & 2.226887 \\
\hline 6 & 3.701730 & 2.190618 & 1.010523 \\
\hline 6 & 4.271003 & 2.181237 & -0.274722 \\
\hline 6 & 5.574143 & 2.636411 & -0.475131 \\
\hline 6 & 6.330447 & 3.105982 & 0.601859 \\
\hline 6 & 5.776333 & 3.116180 & 1.882569 \\
\hline 6 & 4.470644 & 2.664293 & 2.084685 \\
\hline
\end{tabular}




$\begin{array}{lrrr}6 & -2.938267 & 3.922962 & -0.890490 \\ 6 & -3.778623 & 3.806954 & -2.013419 \\ 6 & -4.523217 & 4.895938 & -2.462012 \\ 6 & -4.452995 & 6.119885 & -1.792554 \\ 6 & -3.631571 & 6.247250 & -0.670950 \\ 6 & -2.881401 & 5.160688 & -0.223742 \\ 6 & -3.748184 & -3.086269 & 0.395507 \\ 6 & -3.999533 & -4.195435 & -0.430145 \\ 6 & -4.991855 & -5.117010 & -0.098447 \\ 6 & -5.741463 & -4.951938 & 1.067712 \\ 6 & -5.496390 & -3.857051 & 1.899225 \\ 6 & -4.513253 & -2.927470 & 1.564253 \\ 6 & 1.988778 & -1.785342 & -1.170391 \\ 8 & 2.154021 & -0.576447 & -1.031766 \\ 6 & 3.088401 & -2.628534 & -1.747219 \\ 6 & 4.407865 & -2.167535 & -1.611967 \\ 6 & 5.466961 & -2.886061 & -2.158171 \\ 6 & 5.220117 & -4.064462 & -2.869531 \\ 6 & 3.910336 & -4.518495 & -3.030651 \\ 6 & 2.848943 & -3.808492 & -2.467716 \\ 1 & -1.376398 & -4.739209 & 0.375971 \\ 1 & 1.213926 & -4.593597 & -0.316004 \\ 1 & -1.166548 & 0.126425 & -0.332813 \\ 1 & -4.972221 & 1.988184 & -0.142777 \\ 1 & -5.282727 & -0.671214 & 0.127719 \\ 1 & 2.234409 & 4.308346 & -0.174281 \\ 1 & -0.234593 & 4.943456 & -1.013614 \\ 1 & 4.134606 & -0.122074 & 2.570075 \\ 1 & 2.837092 & -2.179379 & 3.719671 \\ 1 & 0.011148 & 0.381866 & 1.784397 \\ 1 & 3.690468 & 1.785954 & -1.103052 \\ 1 & 6.002699 & 2.617621 & -1.473962 \\ 1 & 7.345511 & 3.460593 & 0.443219 \\ 1 & & 3.486500 & 2.725721 \\ 1 & & & \\ 1 & & & \\ 1 & & & \\ 1 & & & \\ 1 & & & \end{array}$




$\begin{array}{lrrr}1 & -5.155718 & 4.789779 & -3.339271 \\ 1 & -5.037511 & 6.967130 & -2.140822 \\ 1 & -3.580289 & 7.192070 & -0.136560 \\ 1 & -2.260594 & 5.255847 & 0.661709 \\ 1 & -3.423613 & -4.317753 & -1.342268 \\ 1 & -5.180634 & -5.963152 & -0.753586 \\ 1 & -6.511523 & -5.673059 & 1.328063 \\ 1 & -6.067684 & -3.728448 & 2.814496 \\ 1 & -4.311416 & -2.087398 & 2.221492 \\ 1 & 4.578749 & -1.241366 & -1.072357 \\ 1 & 6.485752 & -2.528106 & -2.035201 \\ 1 & 6.046516 & -4.623085 & -3.301321 \\ 1 & 3.713175 & -5.424496 & -3.597492 \\ 1 & 1.831463 & -4.158487 & -2.610015\end{array}$


19,sp-22,24H-3 computational data (B3LYP/6-31G*)

Energy: -2064.17563972 a.u.

\begin{tabular}{|c|c|c|c|}
\hline \multirow{2}{*}{$\begin{array}{l}\text { Atomic } \\
\text { Number }\end{array}$} & \multicolumn{3}{|c|}{ Coordinates (Angstroms) } \\
\hline & $\mathrm{X}$ & $\mathrm{Y}$ & $\mathrm{Z}$ \\
\hline 6 & -0.855500 & -3.813590 & -0.076986 \\
\hline 6 & 0.472729 & -3.742663 & -0.492324 \\
\hline 6 & 0.712803 & -2.419091 & -0.913627 \\
\hline 7 & -0.449071 & -1.726417 & -0.762748 \\
\hline 6 & -1.430927 & -2.529650 & -0.247239 \\
\hline 6 & -2.754516 & -2.069965 & 0.057357 \\
\hline 6 & -3.105010 & -0.715982 & 0.030251 \\
\hline 7 & -2.178550 & 0.288970 & -0.159236 \\
\hline 6 & -2.860194 & 1.440717 & -0.201818 \\
\hline 6 & -4.291737 & 1.202728 & -0.050607 \\
\hline 6 & -4.440910 & -0.142159 & 0.108888 \\
\hline 6 & -2.211306 & 2.724077 & -0.342994 \\
\hline 6 & -0.851451 & 2.883255 & -0.091769 \\
\hline 7 & -0.031172 & 1.936210 & 0.488910 \\
\hline 6 & 1.260454 & 2.368229 & 0.561888 \\
\hline 6 & 1.278730 & 3.697503 & -0.017745 \\
\hline 6 & 0.007615 & 4.010760 & -0.397826 \\
\hline 6 & 2.316102 & 1.636243 & 1.128784 \\
\hline 6 & 2.078622 & 0.375883 & 1.749681 \\
\hline 6 & 3.153195 & -0.505865 & 2.315473 \\
\hline 6 & 2.539628 & -1.605348 & 2.769068 \\
\hline 6 & 1.063672 & -1.424022 & 2.494041 \\
\hline 8 & 0.177510 & -2.217881 & 2.767158 \\
\hline 7 & 0.877488 & -0.180240 & 1.865847 \\
\hline 6 & 3.698200 & 2.184914 & 1.039244 \\
\hline 6 & 4.295308 & 2.400160 & -0.215754 \\
\hline 6 & 5.589460 & 2.913395 & -0.311944 \\
\hline 6 & 6.314586 & 3.213213 & 0.843142 \\
\hline 6 & 5.736061 & 2.997722 & 2.095605 \\
\hline 6 & 4.439435 & 2.491611 & 2.192617 \\
\hline
\end{tabular}




\begin{tabular}{|c|c|c|c|}
\hline & -3.017961 & 3.894543 & -0.768538 \\
\hline & -3.877724 & 3.807124 & -1.879627 \\
\hline & -4.634944 & 4.904133 & -2.285577 \\
\hline & -4.554965 & 6.111900 & -1.588322 \\
\hline & -3.713401 & 6.212348 & -0.479114 \\
\hline & -2.955185 & 5.115423 & -0.071690 \\
\hline & -3.775880 & -3.089027 & 0.407111 \\
\hline & -4.013269 & -4.192507 & -0.431915 \\
\hline & -4.982531 & -5.139053 & -0.104736 \\
\hline & -5.722632 & -5.008404 & 1.072348 \\
\hline & -5.489685 & -3.922807 & 1.918901 \\
\hline 6 & -4.528034 & -2.968943 & 1.589173 \\
\hline & 1.910348 & -1.702648 & -1.381332 \\
\hline & 1.893601 & -0.471613 & -1.450441 \\
\hline D & 3.145403 & -2.464534 & -1.748314 \\
\hline 6 & 4.384833 & -1.839929 & -1.537814 \\
\hline & 5.567307 & -2.485312 & -1.887375 \\
\hline U & 5.526286 & -3.752643 & -2.476367 \\
\hline 6 & 4.297316 & -4.369855 & -2.714308 \\
\hline 6 & 3.111363 & -3.732449 & -2.348084 \\
\hline 1 & -1.361694 & -4.672319 & 0.338221 \\
\hline 1 & 1.203191 & -4.537022 & -0.458800 \\
\hline 1 & -0.561047 & -0.716922 & -0.846610 \\
\hline & -5.064312 & 1.959408 & -0.041668 \\
\hline 1 & -5.362433 & -0.692416 & 0.238581 \\
\hline 1 & -0.307185 & 1.017094 & 0.845650 \\
\hline 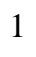 & 2.163203 & 4.310382 & -0.113546 \\
\hline & -0.325193 & 4.917504 & -0.881676 \\
\hline 1 & 4.208589 & -0.267279 & 2.322654 \\
\hline 1 & 2.960570 & -2.482203 & 3.243890 \\
\hline 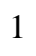 & 3.740495 & 2.136788 & -1.112135 \\
\hline 1 & 6.034478 & 3.070233 & -1.291237 \\
\hline & 7.323402 & 3.610391 & 0.768328 \\
\hline & 6.291556 & 3.231746 & 3.000169 \\
\hline & 3.986380 & 2.343630 & 3.169336 \\
\hline & -3.932789 & 2.875502 & -2.434960 \\
\hline
\end{tabular}




$\begin{array}{lrrr}1 & -5.284416 & 4.817406 & -3.152635 \\ 1 & -5.147488 & 6.966112 & -1.904563 \\ 1 & -3.654413 & 7.143293 & 0.078558 \\ 1 & -2.324587 & 5.190205 & 0.809251 \\ 1 & -3.447206 & -4.290517 & -1.353290 \\ 1 & -5.161833 & -5.978053 & -0.771722 \\ 1 & -6.473989 & -5.750075 & 1.329710 \\ 1 & -6.052173 & -3.821161 & 2.843027 \\ 1 & -4.330831 & -2.137691 & 2.258656 \\ 1 & 4.399578 & -0.849435 & -1.094378 \\ 1 & 6.522596 & -2.000072 & -1.705931 \\ 1 & 6.449589 & -4.253521 & -2.755454 \\ 1 & 4.260244 & -5.345914 & -3.190565 \\ 1 & 2.157816 & -4.205407 & -2.558979\end{array}$


19,ap-22,24H-3 computational data (B3LYP/6-31G*)

Energy: -2064.17194734 a.u.

\begin{tabular}{|c|c|c|c|}
\hline \multirow{3}{*}{$\begin{array}{l}\text { Atomic } \\
\text { Number } \\
6\end{array}$} & \multicolumn{3}{|c|}{ Coordinates (Angstroms) } \\
\hline & $X$ & $\mathrm{Y}$ & $\mathrm{Z}$ \\
\hline & 2.936895 & -3.180476 & 0.707504 \\
\hline 6 & 1.829047 & -3.754350 & 1.322307 \\
\hline 6 & 0.893530 & -2.730745 & 1.552481 \\
\hline 7 & 1.433711 & -1.563676 & 1.098544 \\
\hline 6 & 2.675599 & -1.795826 & 0.554251 \\
\hline 6 & 3.468813 & -0.764369 & -0.033953 \\
\hline 6 & 2.990293 & 0.536898 & -0.243043 \\
\hline 7 & 1.669626 & 0.895837 & -0.049426 \\
\hline 6 & 1.598347 & 2.218673 & -0.261621 \\
\hline 6 & 2.907330 & 2.764522 & -0.587382 \\
\hline 6 & 3.771654 & 1.709547 & -0.593793 \\
\hline 6 & 0.359219 & 2.964038 & -0.231550 \\
\hline 6 & -0.878217 & 2.349152 & -0.376847 \\
\hline 7 & -1.078059 & 1.018050 & -0.696886 \\
\hline 6 & -2.406453 & 0.727179 & -0.821536 \\
\hline 6 & -3.121081 & 1.955225 & -0.530446 \\
\hline 6 & -2.203161 & 2.926326 & -0.262100 \\
\hline 6 & -2.916112 & -0.526565 & -1.205413 \\
\hline 6 & -2.031884 & -1.596386 & -1.526344 \\
\hline 6 & -2.466148 & -2.977653 & -1.919489 \\
\hline 6 & -1.348082 & -3.677300 & -2.145803 \\
\hline 6 & -0.189925 & -2.740297 & -1.897106 \\
\hline 8 & 0.994204 & -3.000252 & -2.034959 \\
\hline 7 & -0.705505 & -1.493606 & -1.493427 \\
\hline 6 & -4.392553 & -0.706572 & -1.302448 \\
\hline 6 & -5.219872 & -0.511984 & -0.182685 \\
\hline 6 & -6.602685 & -0.671297 & -0.277039 \\
\hline 6 & -7.187635 & -1.036991 & -1.490765 \\
\hline 6 & -6.378737 & -1.238401 & -2.610705 \\
\hline 6 & -4.996993 & -1.070136 & -2.518520 \\
\hline
\end{tabular}




\begin{tabular}{|c|c|c|c|}
\hline & 0.430215 & 4.439772 & -0.073944 \\
\hline & 1.193236 & 5.006210 & 0.964002 \\
\hline & 1.266234 & 6.388345 & 1.125748 \\
\hline & 0.585861 & 7.236146 & 0.248549 \\
\hline & -0.165305 & 6.690070 & -0.793588 \\
\hline & -0.241059 & 5.306887 & -0.955010 \\
\hline & 4.864019 & -1.098846 & -0.417894 \\
\hline & 5.753372 & -1.672865 & 0.507762 \\
\hline & 7.064448 & -1.972427 & 0.141360 \\
\hline & 7.506105 & -1.717079 & -1.158590 \\
\hline 6 & 6.629754 & -1.156085 & -2.089448 \\
\hline 6 & 5.321252 & -0.844869 & -1.722911 \\
\hline 6 & -0.438761 & -2.901433 & 2.164787 \\
\hline 8 & -0.915955 & -4.032596 & 2.229344 \\
\hline 6 & -1.200440 & -1.731140 & 2.713489 \\
\hline 6 & -2.593374 & -1.869016 & 2.828925 \\
\hline 6 & -3.361141 & -0.849074 & 3.382735 \\
\hline 6 & -2.746495 & 0.321111 & 3.840571 \\
\hline 6 & -1.361499 & 0.459953 & 3.747722 \\
\hline 6 & -0.590309 & -0.559344 & 3.187312 \\
\hline 1 & 3.832750 & -3.684341 & 0.376433 \\
\hline 1 & 1.676041 & -4.789203 & 1.590893 \\
\hline 1 & 0.971148 & -0.668683 & 0.926578 \\
\hline 1 & 3.125958 & 3.800284 & -0.808084 \\
\hline 1 & 4.835053 & 1.729659 & -0.787188 \\
\hline 1 & -0.360717 & 0.312600 & -0.896426 \\
\hline 1 & -4.196681 & 2.053226 & -0.538565 \\
\hline 1 & -2.400712 & 3.953332 & 0.008243 \\
\hline 1 & -3.494452 & -3.307652 & -1.982629 \\
\hline 1 & -1.232082 & -4.710925 & -2.444406 \\
\hline 1 & -4.765575 & -0.253583 & 0.769821 \\
\hline 1 & -7.223214 & -0.519896 & 0.602621 \\
\hline 1 & -8.264317 & -1.164923 & -1.563104 \\
\hline 1 & -6.824141 & -1.517451 & -3.562084 \\
\hline 1 & -4.375458 & -1.206282 & -3.399396 \\
\hline & 1.717583 & 4.351177 & 1.653581 \\
\hline
\end{tabular}




$\begin{array}{lrrr}1 & 1.853082 & 6.804061 & 1.940534 \\ 1 & 0.645988 & 8.313929 & 0.372932 \\ 1 & -0.685334 & 7.341589 & -1.490862 \\ 1 & -0.803246 & 4.888564 & -1.784569 \\ 1 & 5.416382 & -1.862164 & 1.522468 \\ 1 & 7.742221 & -2.403487 & 0.873240 \\ 1 & 8.526555 & -1.956763 & -1.444997 \\ 1 & 6.962453 & -0.965631 & -3.106168 \\ 1 & 4.634040 & -0.427419 & -2.452024 \\ 1 & -3.049519 & -2.791280 & 2.484727 \\ 1 & -4.438193 & -0.968006 & 3.468062 \\ 1 & -3.345077 & 1.116386 & 4.276977 \\ 1 & -0.877137 & 1.359030 & 4.118436 \\ 1 & 0.488381 & -0.450760 & 3.151070\end{array}$

